\title{
Pilot Study: Effects of Acupuncture on the Muscle Cramps of Liver Cirrhosis Patients
}

\author{
Seung-mo Kim¹, Min-ah Kwak ${ }^{1}$, Jeong-hyun Joo', Kyung-soon Kim¹, Im-hee Shin², Chang-hyeong Lee ${ }^{3}$ \\ 'Dept. of Internal Medicine, College of Korean Medicine, Daegu Haany University \\ ${ }^{2}$ Dept. of Medical Statistics, School of Medicine, Catholic University of Daegu \\ ${ }^{3}$ Dept. of Internal Medicine, School of Medicine, Catholic University of Daegu
}

\section{Pilot Study: Effects of Acupuncture on the Muscle Cramps of Liver Cirrhosis Patients}

\author{
Seung-mo Kim¹, Min-ah Kwak ${ }^{1}$, Jeong-hyun Joo', Kyung-soon Kim', Im-hee Shin², Chang-hyeong Lee ${ }^{3}$ \\ 'Dept. of Internal Medicine, College of Korean Medicine, Daegu Haany University \\ ${ }^{2}$ Dept. of Medical Statistics, School of Medicine, Catholic University of Daegu \\ ${ }^{3}$ Dept. of Internal Medicine, School of Medicine, Catholic University of Daegu
}

\begin{abstract}
Objective: The purpose of this clinical trial is to examine the efficacy and safety of electroacupuncture on liver cirrhosis patients suffering from muscle cramps.

Methods: A total of 14 liver cirrhosis patients with muscle cramps were treated by electroacupuncture three times a week for four weeks (12 times total). The electrical stimulation was a frequency of $100 \mathrm{~Hz}$. Evaluation of the muscle cramps was measured by a questionnaire of subjective symptoms. The patients' condition was evaluated four times over a period of eight weeks. We also assessed the deterioration of liver disease using the Child-Pugh score and blood tests.

Results: This study proves that four weeks of electroacupuncture treatment is effective in reducing the frequency of muscle cramps and that the effects of electroacupuncture treatment lasted during another four-week follow-up period after treatment.

Conclusions: This study suggests that electroacupuncture treatment is beneficial for improving muscle cramps in liver cirrhosis patients $(p=0.000)$. Electroacupuncture is thought to be a safe treatment for liver cirrhosis patients with muscle cramps without contributing to the worsening of liver function. Further study with a larger sample size is needed to confirm our findings.
\end{abstract}

Key words: liver cirrhosis, muscle cramp, electroacupuncture

\section{Introduction}

Muscle cramps mean involuntary muscle contractions

· 투고일: 2018.03.15, 심사일: 2018.06.29, 게재확정일: 2018.06.28

- Corresponding author: Chang-hyeong Lee Dept. of Internal Medicine, School of Medicine, Catholic University of Daegu, 3056-6 Daemyeong 4-dong, Nam-gu, Daegu, Korea

TEL: +82-53-650-4050 FAX: +82-53-628-4050 E-mail: occ@dhu.ac.kr including pain. Muscle cramp is not life-threatening, but affects the patients' quality of life. It is a condition that normally occurs relatively in liver cirrhosis patients and recognized as a side effect of diuretics ${ }^{1}$.

The aetiology of chronic liver disease is variable. Over $70 \%$ of people with chronic liver disease report painful muscle cramps and a causal relation ship rather than a simple association has been described $^{2.3}$. 
Since 1986 Konokoff's report, muscle cramps began to be accepted into the neuromuscular symptoms of liver cirrhosis ${ }^{4}$. After that, researches on the treatment and related factors of muscle cramps $^{5,6}$ were being accomplished in various ways.

The quinine drug family helps to treat symptoms of muscle cramps, but it is unapproved because of serious side effects?. Another treatment including antiepileptics, calcium channel blockers, and various vitamins, minerals supplements have been studied to treat symptoms of muscle cramps and research shows some are not helpful or have adverse effects such as lightheadedness, nausea, and diarrhea ${ }^{6.7}$.

The electroacupuncture therapy is a treatment that gives the electrical stimulation utilizing a variety of voltage and frequency to the needle, gives a strong stimulus than a simple acupuncture therapy. In the domestic study showed electroacupuncture is alleviating on many diseases such as pain ${ }^{8.9}$, stroke ${ }^{10}$, erectile dysfunction ${ }^{11}$, nausea and vomiting ${ }^{12}$, depression ${ }^{13}$, gastric motility ${ }^{14}$, obesity ${ }^{15}$ etc. Electroacupuncture treatment has been reported to be effective in muscle spasticity and cramps of stroke patients ${ }^{16}$ and protective effect in rat with $\mathrm{CCl}_{4}$-induced liver injury ${ }^{17}$.

Therefore, electroacupuncture treatment has been thought to be effective on muscle cramps of liver cirrhosis patients, this study was designed to evaluate the effects of electroacupuncture treatment for the patients who complain muscle cramps diagnosed of liver cirrhosis.

\section{Methods}

\section{Participant recruitment}

Sixteen participants were enrolled in the clinical trial between July 4, 2013 and December 31, 2013.
Participants were informed of the purpose, method, anticipated adverse dangers and discomforts, disclosure of personal information, compensation, and right to discontinue the clinical trial. All patients signed an agreement to enter at one's own will. This study was approved by the Institutional Review Board of Daegu Catholic University Hospital (approved number : MBCR-13-009). Participants were recruited via a recruitment notice on the web site or a poster. The required sample size was estimated to be a total 14 participants, considering a drop-out rate of $20 \%$.

\section{Inclusion and Exclusion Criteria}

Inclusion criteria were as follows: participants (1) age 18 years or over who complain of muscle cramps was diagnosed of cirrhosis and the frequency of true cramps was at least once a week, regardless of part of cramps; (2) serum bilirubin lever $3 \mathrm{mg} / \mathrm{dL}$ or lower; (3) serum aspartate aminotransferase (AST), alanine aminotransferase (ALT) $100 \mathrm{IU} / \mathrm{L}$ or lower; (4) serum creatinine $1.5 \mathrm{mg} / \mathrm{dL}$ or lower; (5) international normalized ratio (INR) 1.5 or lower. The participants were able to follow-up for the duration of the study, accord with the washout criteria (muscle cramps induce or inhibit drugs a week administered limit) and agreed to keep the restriction rule during the study.

Exclusion criteria were as follows: participants (1) have disease associated with muscle cramps, such as diabetes mellitus, vascular occlusive disease, peripheral neuropathy, phlebitis syndrome and thyroid disease; and (2) neutropenia (absolute neutrophill count $500 / \mathrm{mm}^{3}$ or less) and (3) moderate or severe thrombocytopenia (platelet count $50,000 / \mathrm{mm}^{3}$ less) and (4) worsening liver cancer symptom such as fever, aggravation of liver function, abdominal aortic 
aneurysms, venous thrombosis and (5) inappropriate in this study confirmed by researchers and (6) childbearing age (Urine HGC positive).

\section{Intervention}

The study protocol involved total 8 weeks, 4 weeks of treatment with 3 acupuncture sessions per week after screening and 4 weeks from the final acupuncture evaluated the efficacy.

Acupuncture was applied at 9 acupuncture points: GV20, EX-HN3 and bilaterally at 7 points including LI11, LI10, LI4, ST36, LR3, KI6 and BL62. The all needles were inserted $25 \mathrm{~mm}$ into the skin and the electrical stimulation was put on the ST36 and LR3 points with a frequency of $100 \mathrm{~Hz}$. The acupuncture needles used were sterile, disposable stainless steel needles $(0.20 \times 40 \mathrm{~mm}$; Dongbang Acupuncture Inc., Chungcheongnam-do, Korea), and total of 16 needles were used.

\section{Outcome measurements}

All participants completed the self-report of muscle cramps questionnaire six times (screening, V5, V8, V11, V14, V15) during 8 weeks. The questionnaire of muscle cramps occurrence frequency included "more than once a day', "less than once a day $\sim$ more than once a week', 'less than a once a week $\sim$ more than once a month', 'less than once a month', 'none'.

\section{Safety evaluation}

To evaluate the safety of acupuncture, body temperature, blood pressure, pulse and body weight of the participants were checked at each visit. Child-Pugh scores and biochemistry tests (Total Bilirubin, AST, ALT, Albumin, blood urea nitrogen (BUN), creatinine $(\mathrm{Cr})$, INR) were performed at visit 1, 14, 15 for patients. Adverse events were examined by the investigator at each visit.

To check for aggravation of liver cirrhosis, patients were checked of hepatic coma, hepatorenal syndrome, hemorrhage from rupture of esophageal and gastric varices, infection/sepsis, spontaneous peritonitis, and liver function failure. For patients with aggravated liver cirrhosis, the investigator decided whether to discontinue the trial and hospitalization.

Compliance was calculated from the attendance rate of more than $80 \%$, and any participant absent for 5 consecutive appointments was regarded as a drop-out. Participants were told to immediately report any adverse events between visits. Any abnormal condition following acupuncture was to be investigated in relation to the clinical trial and followed-up.

\section{Statistical analysis}

The statistical methods used were based on Statistics Guidelines for Clinical Trials (KFDA, 2000). The data was analyzed using per pro approaches. Statistical analyses were carried out using the program SPSS Win Ver 19.0. Repeated measure ANOVA analysis was used to analyze the frequency of muscle cramps, and significance was set as $p=0.05$.

\section{Results}

\section{Baseline characteristics}

Sixteen patients were screened for eligibility, one of them was screening failure and other one dropped-out, so fourteen patients completed the study. Except the LC-A009 who had only twice acupuncture treatment and dropped-out, all patients have shown over $90 \%$ compliance (Fig. 1).

Fourteen completed participants consisted of 4 men and 10 women patients, the mean age was 59.64 years old (Table 1 ). 


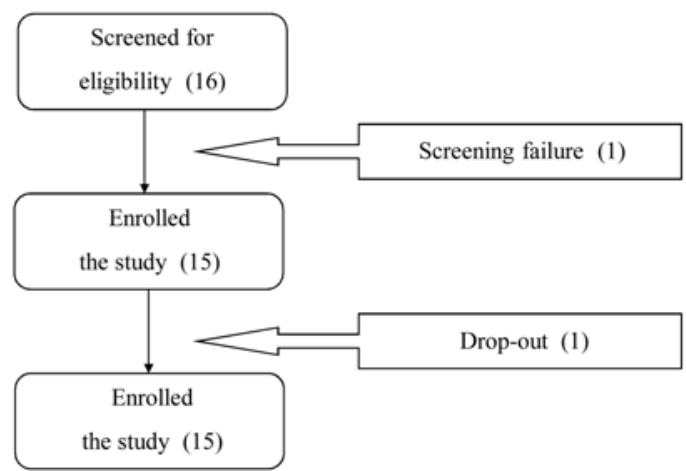

Fig. 1. Study flow chart.

Table 1. Baseline Characteristics of Patients

\begin{tabular}{|c|c|c|}
\hline \multicolumn{2}{|c|}{ Variable } & $\mathrm{n}(\%)$ or mean (S.D.) \\
\hline \multirow{2}{*}{ Sex, n (\%) } & Male & $4(28.6)$ \\
\hline & Female & $10(71.4)$ \\
\hline Age, meal & (S.D.) & $59.64(6.428)$ \\
\hline
\end{tabular}

\section{Frequency of muscle cramp}

All participants completed the self-report of muscle cramps questionnaire 6 times (screening, V5, V8, V11, V14, V15) during the study.

Looking at the change of muscle cramps occurrence frequency in the questionnaire, the number of patients have occurred 'more than once a day' or 'less than once a day $\sim$ more than once a week' has reduced to $14,8,4,2$ and the proportion of patients has decreased 100, 57.1, 28.6, 14.3\%. The number of patients have occurred 'none' or 'less than once a week $\sim$ more than once a month' or 'less than once a month' has increased 0, 6, 10, 12 and the proportion of patients has increased 0, 42.9, 71.4, 85.7\%.

The electroacupuncture treatment is effective in reducing the frequency of muscle cramps $(p=0.000)$ (Table 2).

Table 2. Change of Muscle Cramps Occurrence Frequency

\begin{tabular}{|c|c|c|c|}
\hline & \multicolumn{2}{|c|}{ Muscle cramps occurrence frequency, n(\%) } & \multirow[b]{2}{*}{ n $\begin{array}{c}\text { Total } \\
(100 \%)\end{array}$} \\
\hline & $\begin{array}{l}\text { 'none or 'less than once a week } \sim \text { more } \\
\text { than once a month' or 'less than once } \\
\text { a month' }\end{array}$ & $\begin{array}{l}\text { 'more than once a day' or 'more than } \\
\text { once a week less than once a day' }\end{array}$ & \\
\hline Screening & $0(0)$ & $14(100)$ & 14 \\
\hline Visit 8 & $6(42.9)$ & $8(57.1)$ & 14 \\
\hline Visit 14 & $10(71.4)$ & $4(28.6)$ & 14 \\
\hline Visit 15 & $12(85.7)$ & $2(14.3)$ & 14 \\
\hline$x^{2}(p-$ value $)$ & \multicolumn{2}{|c|}{$21.000^{\dagger}(0.000)^{*}$} & - \\
\hline
\end{tabular}

\section{Safety}

To evaluate the safety of acupuncture, we performed liver function tests (Table 3). There were no significant differences in T-Bilirubin, AST, ALT, Albumin, BUN, Cr, INR (Table 3). Furthermore, aggravation of liver cirrhosis and primary complications (hepatic encephalopathy, hepato-renal syndrome, esophageal/gastric varices bleeding, infection/sepsis, spontaneous peritonitis) were not observed. All patients showed good compliance. Adverse events were checked every visit during the course of trial, and no adverse events were observed in the course of study in any of the fourteen patients. 
Table 3. Mean Change of Child Score and Blood Composition

\begin{tabular}{ccccc}
\multirow{2}{*}{ Variable } & \multicolumn{3}{c}{ time, Mean (S.D.) } & F (p-value) \\
\cline { 2 - 4 } & Screening & V14 & V15 & \\
\hline Child score & $5.29(0.61)$ & $5.36(0.74)$ & $5.36(0.63)$ & $0.481(0.623)$ \\
T-bil & $1.33(0.49)$ & $1.32(0.58)$ & $1.35(0.49)$ & $0.113(0.894)$ \\
AST & $37.79(15.52)$ & $50.21(24.16)$ & $51.36(24)$ & $6.316(0.006)^{*}$ \\
ALT & $24.57(7.88)$ & $27.5(12.31)$ & $26.64(11.43)$ & $1)(2), 3)^{\dagger}$ \\
Alb & $3.82(0.41)$ & $3.85(0.37)$ & $3.83(0.45)$ & $1.039(0.368)$ \\
INR & $1.17(0.12)$ & $1.12(0.12)$ & $1.13(0.12)$ & $0.086(0.918)$ \\
BUN & $13.21(4.28)$ & $21.11(24.27)$ & $14.71(6.1)$ & $4.025(0.030)^{*}$ \\
Cr & $0.8(0.23)$ & $0.79(0.27)$ & $0.82(0.28)$ & $1.291(0.292)$ \\
\hline
\end{tabular}

* : statistically significant with $\mathrm{p}<0.05$

$\dagger$ : multiple comparison result by contrast

T-bil : total bilirubin

AST : aspartate aminotransferase

ALT : alanine aminotransferase

Alb : albumin

INR : international normalized ratio

BUN : blood urea nitrogen

$\mathrm{Cr}$ : creatinine

\section{Discussion}

Acupuncture and electroacupunture are somewhat effective treatment for liver cirrhosis patients experiencing muscle cramps and that would help improving the quality of life. So this study is a clinical trial to demonstrate the effect of electroacupuncture treatment for liver cirrhosis with muscle cramps.

Sixteen patients were screened for eligibility, one of them was screening failure and other one dropped-out, so fourteen patients completed the study.

This study proves the during 4 weeks of electroacupuncture treatment is effective in reducing the frequency of muscle cramps and the effects of acupuncture treatment also lasting during 4 weeks follow-up period (Fig. 2).

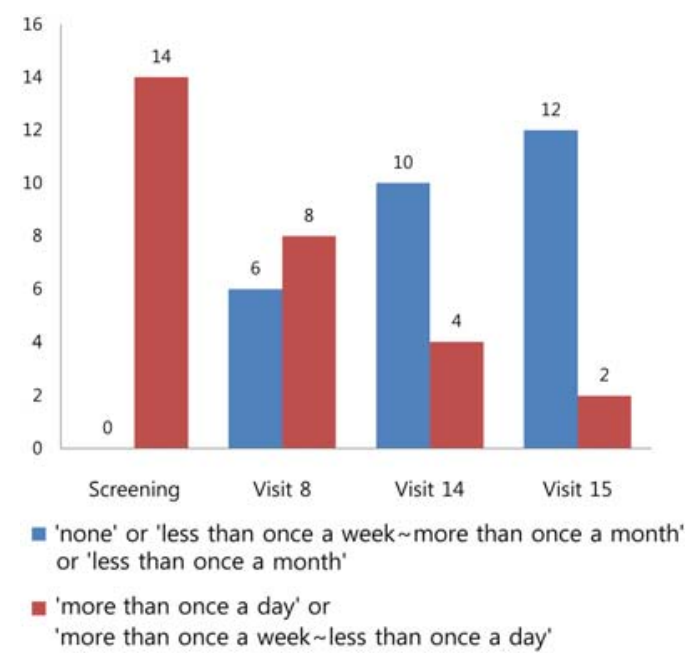

Fig. 2. Change of muscle cramps occurrence frequency.

The electroacupuncture therapy is a treatment that gives the electrical stimulation utilizing a variety of voltage and frequency to the needle, give a strong 
stimulus than a simple acupuncture therapy and studies of electroacupuncture have been made on a wide range of diseases ${ }^{8-15}$.

Some studies show that electroacupuncture is effective way to treat diseases, especially muscle cramps caused by spinal cord injury ${ }^{16}$ undergoing hemodialysis ${ }^{17}$ and $\mathrm{Heo}^{18}$ et al. reported the effect of electroacupuncture at Yanglingquan (GB34) on $\mathrm{CCl}_{4}$-induced liver damage in rats.

The acupuncture points used in this study were selected based on previous studies ${ }^{19-23} \cdot 16$ acupuncture points are located in lower limbs and arms, which occur muscle cramps frequently. As acupuncture location, extremities are effective on the relieving pain, calming and activating cerebral blood-flow. Each acupoints has the following effects. Both LI4 and LR $3^{19}$ points, namely 'four gates', are clinically used in stress-related symptoms by regulating the flow of blood and qi. The LI4 is the main acupoint of the qi and the LR3 is the other main acupoint of blood. In addition, LR3 acupoint is in the liver meridian and has effects on the hepatitis, cholecystisis and convulsion of epilepsy caused by liver diseases. GV20 ${ }^{20}$ acupoint located the highest position of our body so it can boosting up our yang-qi. GV20 has effects on dementia, insomnia, epilepsy and mental diseases. EX-HN3 ${ }^{21}$ point has a calming effects such as insomnia, headache and vertigo. This two acupoints located in our head and increases the head blood circulation, thus could treat head diseases. KI6 and BL62 ${ }^{22}$ acupoints, which are located on the ankle joint, could balance our yin and yang-qi and treat our sleep disorders. ST36, LI10 and LI11 acupoints are located on the limbs, which occur muscle cramps frequently. ST $36^{23}$ is the main acupoint of gastrointestinal diseases and strengthening the body's resistance and restoring our vital energy. LI10 and LI11 are located on the upper extremities so they are used on numbness, pain and motor impairment of the hand (Table 4).

Table 4. Acupuncture Points Used in this Study

\begin{tabular}{|c|c|c|}
\hline Points & Meridian & Location \\
\hline GV20 & Governing vessel & Midway on a line connecting the apex of both ears \\
\hline EX-HN3 & Extra points & On the forehead, at the midpoint between the two medial ends of the eyebrow \\
\hline LI11 & Large intestine & $\begin{array}{l}\text { When the elbow is flexed, the point is in the midpoint between the lateral } \\
\text { end of the transverse cubical crease and the lateral epicondyle of the humerus. }\end{array}$ \\
\hline LI10 & Large intestine & On the outer surface of the forearm and 2 cun* below LI11 \\
\hline LI4 & Large intestine & $\begin{array}{l}\text { On the dorsum of the hand, midway between the } 1^{\text {st }} \text { and } 2^{\text {nd }} \text { metacarpal bones, } \\
\text { approximately in the middle of the } 2^{\text {nd }} \text { metacarpal bone on the radial side }\end{array}$ \\
\hline ST36 & Stomach & 3 cun* below Dubi, on finger-breadth from the anterior crest of the tibia \\
\hline LR3 & Liver & $\begin{array}{l}\text { On the dorsum of the foot, in the depression distal on the junction of the } 1^{\text {st }} \\
\text { and } 2^{\text {nd }} \text { metatarsal bones }\end{array}$ \\
\hline KI6 & Kidney & 1 cun* directly below the tip of the medial malleolus \\
\hline BL62 & Bladder & $\begin{array}{l}\text { On the lateral side of the foot, in the depression below the lateral malleolus } \\
\text { and posterior to the peroneal tendons }\end{array}$ \\
\hline
\end{tabular}


We attempted to minimize interaction with patients, since acupuncture treatment could foster a relationship between patients and practitioners, consequently result in a placebo effect ${ }^{24,25}$. One study showed more improvement of irritable bowel syndrome when permitted to have a relationship with the practitioner ${ }^{26}$. Therefore, we restricted talk with patients except for advice agreed upon before treatment. There were no evident adverse events, such as subcutaneous bleeding, following acupuncture. 4 weeks of acupuncture treatment improved the muscle cramps of liver cirrhosis patients. Furthermore, there were no side effects such as aggravation of liver function and complications associated with liver cirrhosis.

Acupuncture may be safe and feasible treatment for the muscle cramps of liver cirrhosis patients. Further retrospective clinical trials using a larger patient cohort will be needed to verify the efficacy and safety of acupuncture for the muscle cramps of liver cirrhosis patients. Also, research using sham control group may be warranted to investigate the effects of acupuncture.

\section{v. Conclusions}

In this study, the patients, who complain of muscle cramps diagnosed with liver cirrhosis and the frequency of true cramps was at least once a week regardless of part of cramps, were treated by electroacupuncture for 4 week. As a result, the frequency of muscle cramps was very significantly reduced.

There was no aggravation of liver function and complications associated with liver cirrhosis according to the electroacupuncture and no evident adverse events by electroacupuncture, such as subcutaneous bleeding. So the fourteen patients were able to complete the study safely.

Therefore clinical trials using a larger patient cohort are required to establish the efficacy and safety of acupuncture for liver disease including liver cancer accompanied by muscle cramps, which is restricted in drug use.

\section{References}

1. Kim GI, Kang SH, Kim JI, Chae GH, Heo WS, Jeong JH, et al. The clinical feature of the muscle cramps and the effects of eperisone hydrochloride in treatment for the muscle cramps of the patients with liver cirrhosis. The Korean Journal of Medicine 2006;70(6):643-9.

2. Minetto MA, Holobar A, Botter A, Farina D. Origin and development of muscle cramps. Exerc Sport Sci Rev 2013;41(1):3-10.

3. Mehta SS, Fallon MB. Muscle cramps in liver disease. Clin Gastroenterol Hepatol 2013;11(11) :1385-91.

4. Konikiff F, Theodor E. Painful muscle cramps : a symptom of liver cirrhosis? $J$ Clin Gastroenterol $1986 ; 8(6): 669-72$.

5. Cho JH, Choi JI, Keum MS, Tak WY, Kweon YO, Kim SK, et al. Muscle Cramps in Liver Cirrhosis : Clinical Features and Related Factors. Korean J Gastroenterol 1999:34(1):76-82.

6. Lee KH, Kim NY, Koh YH, Koh JJ, Oh JH, Lee CG, et al. Muscle Cramps in Patients with Chronic Liver Disease. Korean $J$ Gastroenterol 1998:32(3):346-54.

7. Hans DK, Ahmir HK, Yuen T. Assessment: Symptomatic treatment for muscle cramps (an evidence-based review). Neurology 2010;74(8) :691-6.

8. Seo DM, Kang SK. Systemic Review: The Study 
Pilot Study: Effects of Acupuncture on the Muscle Cramps of Liver Cirrhosis Patients

on Electroacupuncture in Pubmed. The $J$ of Korean Acupuncture \& Moxibustion Society 2002; 19(3) :168-79.

9. Coura LE, Manoel CH, Poffo R, Bedin A, Westphal GA. Randomized controlled study of preoperative electroacupuncture for post operative pain control after cardiac surgery. Acupunct Med 2011;29(1):16-20.

10. Wang XB, Chen J, Li Tj, Tao J, Chen LD, He J, et al. Effect of electroacupuncture in different frequencies on electromyography and ambulation in stroke patients with lower extremity spasticity : a randomized controlled study. Zhongguo Zhen Jiu 2011;31(7):580-4.

11. Dorey G. Conservative treatment of electile dysfunction. 3. : Literature review. Br J Nurs 2000:9(13):859-63.

12. Lee A, Done ML. The use of non-pharmacologic techniques to prevent post operative nausea and vomiting: a meta-analysis. Anesth Analog 1999:88(6) :1362-9.

13. Luo H, Meng F, Jia Y, Zhao X. Clinical research on the therapeutic effect of the electroacupuncture treatment in patients with depression. Psychiatry Clin Neurosci 1998:52 Suppl:S338-40.

14. Lin X, Liang J, Ren J, Mu F, Zhang M, Chen JD. Electrical stimulation of acupuncture points enhances gastric myoeletrical activity in humans. Am J Gastroenteral 1997;92(9) :1527-30.

15. Hwang DS, Lee YJ, Lee CH, Kim YS, Jang JB, Lee KS. The Efficacy of Low Frequency Electroacupuncture Therapy for Weight Loss in Obese Korean Women. The $J$ of Oriental Obstetrics \& Gynecology 2009:22(2):140-50.

16. Moon JS, Lee SH, Kim JE, Kim BY, Choi SM. A Survey on the Use of Electroacupuncture by Traditional Korean Medicine Doctor. The $J$ of
Korean Acupuncture \& Moxibustion Society 2011;28(6):53-68.

17. Mohmadi K, Shahgholian N, Valiani M, Mardanparvar $\mathrm{H}$. The effect of acupressure on muscle cramps in patients undergoing hemodialysis. Iran $J$ Nurs Midwifery Res 2016;21(6):557-61.

18. Heo YK, Lee H. A Study on the Effect of High Frequency Electroacupuncture at Yanglingquan (GB34) on $\mathrm{CCl}_{4}$-intoxicated Rats. The Korean Journal of Meridian \& Acupoint 2009:26(1):111-23.

19. Park TG, Kim YI, Hong KE, Yim YK, Lee H, Lee BR. A study on brain activity induced by electro-acupuncture on Taechung(LR3) and Hapkok(LI4) using functional Magnetic Resonance Imaging. The Korean Journal of Meridian \& Acupoint 2004;21(2) :29-46.

20. Song HC, Bom HS, Kang HJ, An SG, Kim SM, Jung HJ, et al. Effect of Acupuncture on Regional Cerebral Blood Flow at Acupoints GV 20, GV. 26, LI. 4, ST. 36, SP. 6 Evaluated by Tc-99m ECD Brain SPECT. Nucl Med Mol Imaging 2000;34(6) : 456-64.

21. Korean Acupuncture \& Moxibustion Society Textbook Compilation Committee. The acupuncture and Moxibustion medicine (下). Seoul: Jipmoondang; 2006, p. 764-1070.

22. Park WS, Lee TY, Kim SY, Leem KG, Yuk $\mathrm{SW}$, Lee $\mathrm{CH}$, et al. The Immediate Effect of Electroacupuncture at the B62 (Shinmaek) K6 (Chohae) on the KKG of Vascular Dementia. The $J$ of Korean Acupuncture \& Moxibustion Society 2001;18(2):67-78.

23. CS Lee, BR Park, JH Seo, Kang HW, Lyu YS, Kim TH, et al. Effects of Hap-Kok(LI4) and Jok-Sam-Lee(ST36) Acupuncture on Cerebral Blood Flow in In-Young(ST9). $J$ of Oriental Neuropsychiatry 2007;18(1):143-51. 
24. Garcia MK, McQuade J, Haddad R, Patel S, Lee R, Yang P, et al. Systematic review of acupuncture in cancer care: a synthesis of the evidence. Journal of clinical oncology : official journal of the American Society of Clinical Oncology 2013;31(7):952-60.

25. Streitberger K, Kleinhenz J. Introducing a placebo needle into acupuncture research. Lancet 1998; 352 (9125):364-5.

26. Kaptchuk TJ, Kelley JM, Conboy LA, Davis RB, Kerr CE, Jacobson EE, et al. Components of placebo effect: randomised controlled trial in patients with irritable bowel syndrome. Bmj 2008;336(7651) :999-1003. 